\title{
THERMOCAPILLARY MONITORING USING PARTICLE IMAGE VELOCIMETRY ASSOCIATED WITH DYNAMIC LASER SPECKLE
}

\section{Ellem Waleska Nascimento da Fonseca Contado}

Federal University of Lavras (UFLA), Department of Engineering (DEG), Campus, P.O. Box 3037, ZIP Code 37200-000, Lavras, MG, Brazil

Corresponding author: ellem.waleska@gmail.com

\section{Roberto Alves Braga Júnior}

Federal University of Lavras (UFLA), Dept. of Automation (DAT), Campus, P.O. Box 3037, ZIP Code 37200-000, Lavras, MG, Brazil

\section{Henrique Coelho Barbosa}

Federal University of Lavras (UFLA), Department of Engineering (DEG), Campus, P.O. Box 3037, ZIP Code 37200-000, Lavras, MG, Brazil

\section{Renan Oliveira Reis}

Federal University of Minas Gerais, Belo Horizonte, MG, Brazil

\section{Radhakrishna Prabhu}

University Robert Gordon, Aberdeen, Scotland, UK

\begin{abstract}
Thermocapillarity is a physical phenomenon used in many industrial processes, mainly in the field of miniaturization. Thermocapillary forces are the base of thermocapillary pumping (TCP), in which a drop of liquid moves through a microchannel or flat surface after temperature gradient occurs. The objective of this work was to study the thermocapillary convection during pumping without inserting external particles, monitored by the Particle Image Velocimetry (PIV) technique. The experiment consisted of a Pasteur tube containing a yellow fluorescein solution $(0.02,0.04,0.06,0.08$, and 0.1 Molar), illuminated by a laser beam of $545 \mathrm{~nm}, 40 \mathrm{~mW}$. After 2 minutes of illumination, the thermocapillary movement occurred at a distance of 0.05 $\mathrm{mm}$ below the meniscus when using a laser beam of $545 \mathrm{~nm}, 3 \mathrm{~mW}$. The images were captured by a charge-coupled device (CCD) camera and processed using the PIV technique. The results showed an internal conversion capacity between the intersystem crossing, vibrational, and relaxation phenomena, also demonstrating the potential for applying the proposed approach. The images presented velocity distribution caused by thermocapillarity. The PIV was a useful tool for convective flow analysis if connected to appropriate image processing and enhancement techniques. In conclusion, the research showed the images with velocity distribution caused by thermocapillarity.
\end{abstract}

Index terms: Termocapillary, Vortex, PIV.

Received: November, 25, 2019 - Accepted: May, 22, 2020

\section{INTRODUCTION}

Thermocapillarity is a physical phenomenon used in many industrial processes, mainly in the field of miniaturization (Maggio, 2000). It is a transfer of mass through an interface between two fluids caused by the surface tension gradient (Sammarco, Mark, 1999). This phenomenon was reported in the $19^{\text {th }}$ century when the "tears of wine" phenomenon was observed on the wall of crystal glass (Wozniak et al., 1994). Thermocapillary pumping (TCP) is a concept based on thermocapillary forces, in which a drop of liquid moves through a microchannel or flat surface after temperature gradient occurs causing a voltage difference on the surface of the drop of liquid, driving this drop to a colder region (Wozniak, Wozniak, 1994). TCP is experimentally studied using particle image velocimetry (PIV) as a liquid crystal marker to 
visualize flow analysis. This method offers the advantage of simultaneously measuring the entire flow field in a selected plane within the fluid at a given time (Kim et al., 2010). The flow field can be the speed field, the temperature distribution, or others.

Fluorescein, a liquid crystal used in TCP, is a hydrocarbon, crystalline, orange molecule, with an approximate molecular weight of 376 $\mathrm{KDa}$, belonging to a class of xanthemic dyes obtained by the condensation of phthalic anhydride with resorcinol in the presence of sulfuric acid (Bayer, 1871). The emission of intense fluorescence, even in diluted solutions, allows fluorescein to function as a marker in liquid solutions. Sodium fluorescein is excited by energy at a wavelength between 465 and 470 (blue wavelength) and emits at a higher wavelength, between 520 and $530 \mathrm{~nm}$ (yellow and green).

In this work, we associated PIV with the speckle phenomenon to monitor the thermocapillary convection during pumping without adopting (inserting) external particles.

\section{MATERIAL AND METHODS}

Figure 1 shows the experimental arrangement, where we added the yellow fluorescein solution in a Pasteur tube in the concentrations of $0.02,0.04,0.06,0.08$, and 0.1 Molar. We then heated the solution by illuminating it with a laser beam of $545 \mathrm{~nm}$ and $40 \mathrm{~mW}$. After two minutes, we observed the thermocapillary movement at $0.05 \mathrm{~mm}$ below the meniscus using a second laser beam of 545 nm with $3 \mathrm{~mW}$.

We captured the images using a chargecoupled device (CCD) camera, processing them through the PIV technique (Soares et al., 2013). We processed the images (Figure 2) by comparison in sequence ( 1 with 2,2 with 3,3 with 4 , etc.).

\section{RESULTS AND DISCUSSION}

Figure 3 demonstrates the sum of the vector modules obtained by PIV for each concentration of fluorescein during heating. The vectors are distributed according to the angular behavior.

There were changes in the angular behavior over heating time for all concentrations of fluorescein employed. This fact is due to the temperature variation in the analyzed region caused by the pumping movement. Wozniak and Wozniak (1990) reported that the convective flow shows movements in increasing temperature gradients. Talmor et al. (2018) observed a highly circulatory convective flow using the PIV technique and generating waves through an electrohydrodynamic pump.

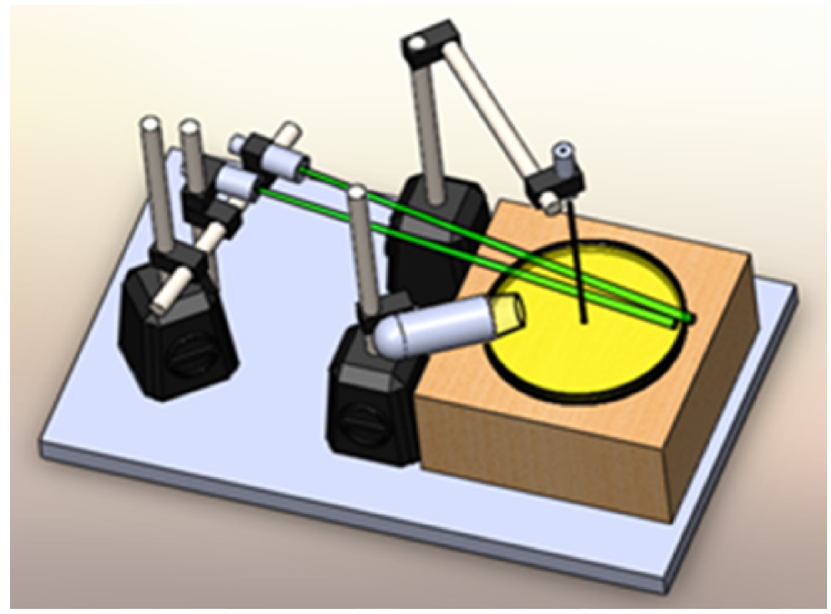

Figure 1: Experimental arrangement of illumination and heating by laser.

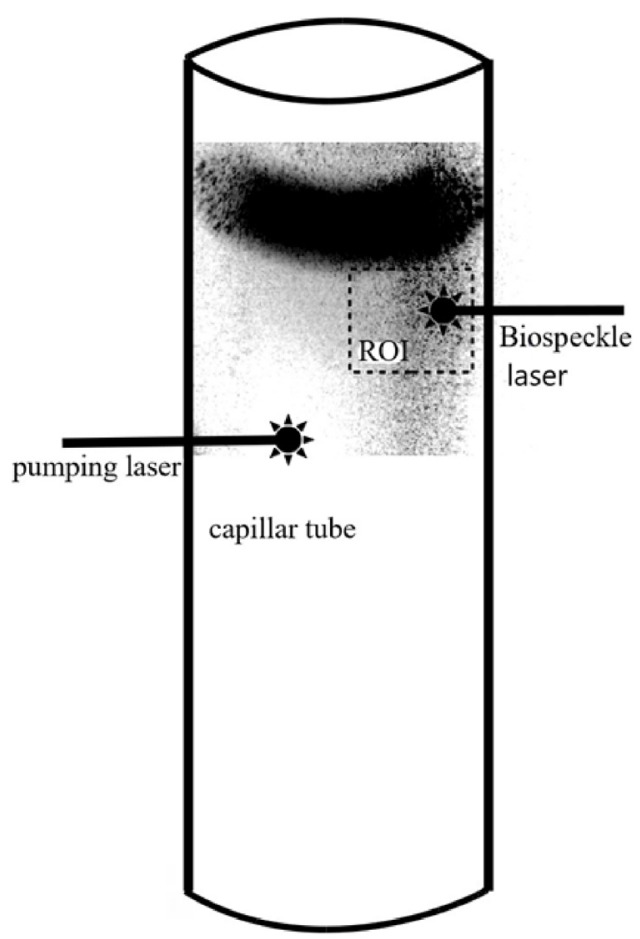

Figure 2: Magnification of the illuminated area. 


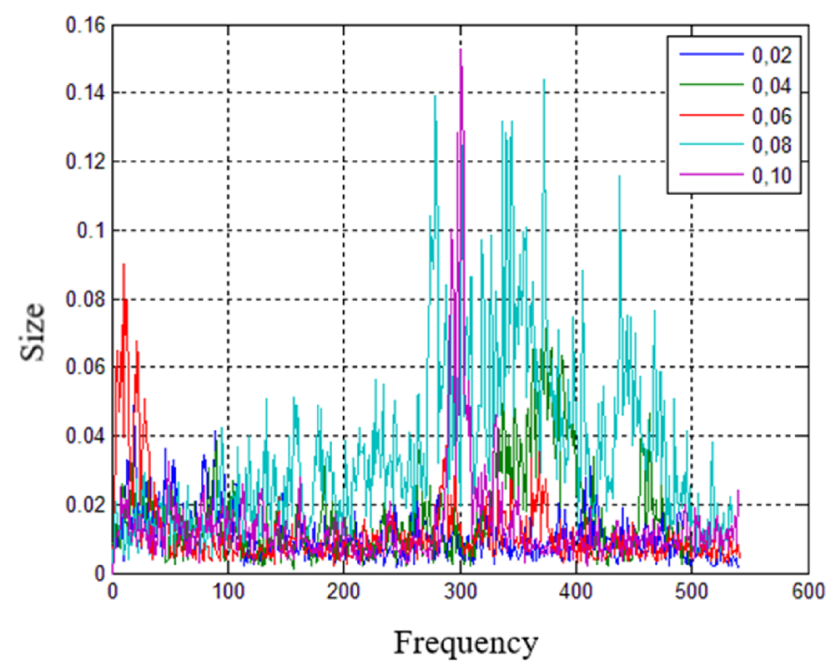

Figure 3: Sum of the vector modules obtained by PIV with all concentrations of fluorescein.

We observed a more significant pumping movement during the time interval from 300 to 400 for all concentrations of fluorescein, which occurs due to the increased pumping movement in the fluid. The variation in surface tension due to the temperature gradient causes convection, which can generate increasing and decreasing angular peaks. In summary, the magnitude of the maximum negative speed is higher than that of the positive speed (Buffone et al., 2005).

We can observe in Figure 3 that the lower concentrations of fluorescein may occur in lower temperatures, thereby causing a low angular behavior since the concentration of $0.08 \mathrm{M}$ can accumulate energy. This can, in turn, result in peaks with angle difference due to the variation of voltage surface caused by the temperature gradient, occurring convection, which can generate increasing and decreasing angular peaks.

Among the different concentrations of fluorescein used, $0.08 \mathrm{M}$ showed the highest pumping movement. Therefore, the research group decided to compare it with the others. The results are presented in Figures $4 a, 4 b, 4 c$, and $4 \mathrm{~d}$. The vectors are distributed according to the angular behavior.

Regarding the sum of vectors expressed in the comparison between $0.08 \mathrm{M}$ and $0.02 \mathrm{M}$, the concentration of 0.08 showed high peaks in the range of 300 to 500 , whereas in $0.02 \mathrm{M}$ remained low (Figure 4a). In the angular behavior of the comparison between $0.08 \mathrm{M}$ and $0.04 \mathrm{M}, 0.04$ $\mathrm{M}$ increased from 300 to 380 , then decreased and, later, peaked at 450 (figure 4b). The angular behavior of $0.08 \mathrm{M}$ remained low when compared to $0.06 \mathrm{M}$ (figure 4c) while spiking at 300 and then remaining low when compared to the concentration of $0.10 \mathrm{M}$ (figure $4 \mathrm{~d}$ ).

When a flow opposite to the flow generated occurs, the result is due to the underlying mechanisms of vortex formation. Therefore, in the case of an opposite flow, we can infer that the flow occurs due to the meniscus heating, while in the dynamic case, it is mostly driven by the surface. According to Talmor et al. (2018), these differences are, once more, due to the confinement of the flow since it has no easy path forward and out of the test section.

The heating of the meniscus causes a convection movement to occur where the heated fluid collides with the cooled fluid. Thus, the pumping movement occurs and, with theincrease in concentration, it was possible to visualize the rise in this pumping fluid movement. According to Ohyama et al. (2007) and Kitahara et al. (2007), most of the techniques used to observe this flow have focused on fluorescent molecules.

The first study that appears in the literature for a qualitative map was performed by Hemayatkhah et al. (2011). They used dye infusion to generate a qualitative map of the flow

The results of the PIV analysis are present in the vector map. The images with the capillary flow pattern show the rotating vortex conducted above the surface tension gradient. This result was possible due to the PIV technique, allowing us to capture all the information of the pumping movement by correlating two images. The speckle technique measures small displacements, pumping movement, with a decrease in interfacial tension and an increase in temperature in the direction of flow in the periphery of the bubble of the heated wall.

With the increase in concentration, we lost control of the vectors and, with a concentration inferior to 0.04 molar, the vectors found were much smaller. When working with 0.06 Molar, we found a noncontinuous pumping movement, while with 0.08 Molar, the pumping movement was continuous.

According to Berejnov and Morozov (2013), the mechanical convection movement appears in a non-uniform heated liquid because its density depends on the temperature. 


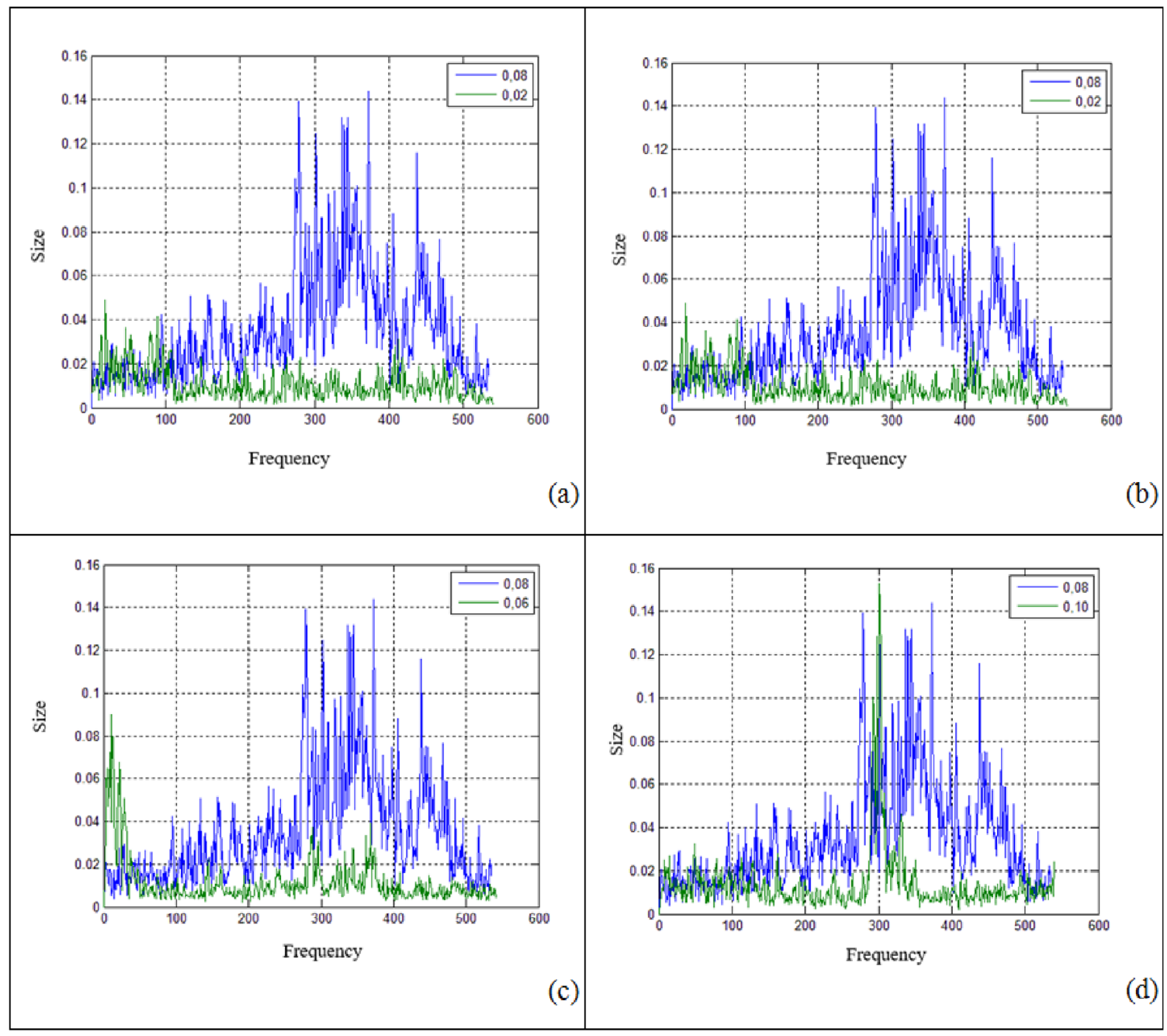

Figure 4: Sum of the vector module obtained by PIV with the concentrations of (a) 0.02 and 0.08 molar, (b) 0.04 and 0.08 molar, (c) 0.06 and 0.08 molar, and (d) 0.08 and 0.1 molar. The blue graph represents the concentration of $0.08 \mathrm{M}$ and the green, its correlation.

The force of the convection is triggered by the surface tension, represented by the vorticity. The vorticity normalized with the convective time scale was initially considered to be highest at the concentration of $0.08 \mathrm{M}$.

\section{CONCLUSIONS}

We demonstrated that particle image velocimetry is a useful tool for analyzing convective flow if connected to appropriate image processing and enhancement techniques. The measuring technique described shows satisfactory results for thermocapillary flows, where the speed is relatively low.

\section{ACKNOWLEDGMENTS}

This work was partially financed by CAPES, FAPEMIG, CNPq, and FINEP in Brazil.

\section{REFERENCES}

BEREJNOV, V.; MOROZOV, I. K. Thermocapillary motion. Physics Fluid Dynamics, arXiv:1303.0243, 2013.

BUFFONE, C.; SEFIANE, K. IR measurements of interfacial temperature during phase change in a confined environment. Experimental Thermal and Fluid Science, 29:65-74, 2004. 
GLONKER, P. S.; NATERER, G. F. Numerical simulation of electrokinetic flow and heat transfer in microchannels with a finite volume method. Numerical Heat Transfer Applications, 49(5):451170, 2006.

HEMAYATKHAH, M.; GHARRAEI, R.; ESMAEILZADEH, E. Flow pattern visualization of liquid film conduction pumping using flush mounted electrode. Experimental Thermal and Fluid Science, 35(6):933-938, 2011.

KAO, Y. S.; KENNING, D. B. R. Thermocapillary flow near a hemispherical bubble on a heated wall. Journal of Fuid Mechanics, 53:715-735, 1972.

KITAHARA, Y.; AOYAGI, K.; OHYAMA, R. An experimental analysis of ionic wind velocity characteristics in a needle-plate electrode system by means of laser-induced phosphorescence". Proceedings of the Annual Conference on Electrical Insulation and Dielectric Phenomena, 529-532, 2007.

KIM, W. et al. Particle image velocimetry of the blood flow in a micro-channel using the confocal laser scanning microscope. Journal of the Optical Society Korea, 14(1):25-30, 2010.

MAGGIO T. S. et al. Thermocapillary pumping of discrete droplet in microfabricated analysis devices. The American Institute of Chemical Engineers Journal, 45(2):350-366,2000 .

OHYAMA, R. et al. Visualization of the local ionic wind profile in a DC corona discharge field by laserinduced phosphorescence emission. Journal of visualization, 10(1):75-82, 2007.

RAAKE, D.; SIEKMANN, J.; CHUN, Ch.-H. Temperature and velocity fields due to surface tension driven flow. Experiments in Fluids, 7:164-172, 1989.

SAMMARCO T. S.; BURNS M. A. Thermocapillary pumping of discrete droplet in microfabricated analysis devices. The American Institute of Chemical Engineers Journal, 45(2):350-366, 2004.

SOARES, R. R. et al. Biospeckle PIV (Paricle Image Velocimetry) for analyzing fluid flow. Flow Measurement and Instumentation, 30:91-98, 2013.

TALMOR, M.; LOUSTE C.; SEYED-YAGOOBI, J. PIV flow field measurements of electrohydrodynamic conduction pumping, Proceedings of Electrostatics Joint Conference, 1-15, 2018.

WOZNIAK, K.; WOZNIAK, G. Particle-imagevelocimetry applied to thermocapillary convection. Experiments in Fluids Springer-Verlag, 10:12-16, 1990.

WOZNIAK, G.; WOZNIAK, K. Buoyancy and thermocapillary flow analysis by the combined use of liquid crystals and PIV. Experiments in Fluids Springer-Verlag, 17:141-146, 1994. 